\title{
Carcinoembryonic antigen-positive pleural effusion in early stage non-small cell lung cancer without pleural infiltration
}

\author{
Njanja Enz ${ }^{1}$, Fernando Fragoso ${ }^{1}$, Alexander Gamrekeli ${ }^{1}$, Frank Lippek ${ }^{2}$, Wolfgang Jungraithmayr ${ }^{1}$ \\ ${ }^{1}$ Department of Thoracic Surgery, ${ }^{2}$ Department of Pathology, Brandenburg Medical School, Neuruppin, Germany \\ Correspondence to: Wolfgang Jungraithmayr, MD, PhD. Department of Thoracic Surgery, Brandenburg Medical School, Fehrbelliner Str. 38, \\ Neuruppin 16816, Germany. Email: wolfgang.jungraithmayr@mhb-fontane.de.
}

\begin{abstract}
Carcinoembryonic antigen (CEA) is a tumor marker for detecting recurrences of adenocarcinomas such as colon cancer. In lung adenocarcinoma, CEA elevation can be found in both serum and malignant pleural effusion. However, CEA elevation in cytologically negative pleural effusion in the presence of adenocarcinoma without pleural infiltration has not been described. We here present the case of an 82-year-old man with incidental early stage adenocarcinoma of the right upper lobe showing CEA elevation in pleural fluid and serum despite negative cytological findings. Due to limited lung reserve the tumor was removed by wide wedge resection, but the visceral pleura was not affected and infiltration of the parietal pleura was ruled out by pleural biopsies. Serum and pleural CEA levels declined postoperatively as measured at 1 and 2 months follow-up. This case shows CEA elevation in serum and pleural fluid in early stage lung adenocarcinoma with negative cytology and no sign of pleural infiltration. Previous research revealed that CEA level in pleural effusion correlates to serum CEA and is significantly higher in adenocarcinoma of the lung than in other lung cancer entities. Firstly, this case suggests that determination of CEA levels can increase the diagnostic sensitivity in cases with cytologically negative pleural effusion suspicious of malignant origin and secondly, it contributes valuable information to the decision whether follow-up of pulmonary nodules or continuative diagnostics such as video-assisted thoracoscopic surgery (VATS) wedge resection is indicated.
\end{abstract}

Keywords: Adenocarcinoma; lung; carcinoembryonic antigen (CEA); pleural effusion

Submitted Oct 29, 2017. Accepted for publication Apr 06, 2018.

doi: $10.21037 /$ jtd.2018.04.111

View this article at: http://dx.doi.org/10.21037/jtd.2018.04.111

\section{Introduction}

Carcinoembryonic antigen (CEA) is a glycoprotein involved in cell adhesion and is usually produced in gastrointestinal tissue during fetal development. Elevated CEA serum levels in adults are found in adenocarcinomas of various origin, thus qualifying CEA as a tumor marker. CEA elevation has been shown for pulmonary adenocarcinoma both in serum and malignant pleural effusion $(1,2)$. However, CEA elevation in cytologically negative pleural effusion accompanying adenocarcinoma without pleural infiltration has not been shown previously.

\section{Case presentation}

We here present an 82-year-old man with an incidental lung adenocarcinoma of the right upper lobe, displaying elevated CEA levels in pleural effusion and serum.

Initial admission was due to progressive dyspnea on exertion and orthopnea 7 months after trauma to the right hemithorax with fracture of rib No. 7 and 8 . The treatment consisted of pain medication.

The patient's history revealed productive cough, movement-induced pain located in the right shoulder, former nicotine abuse until 2008 (40 packyears) and no B 


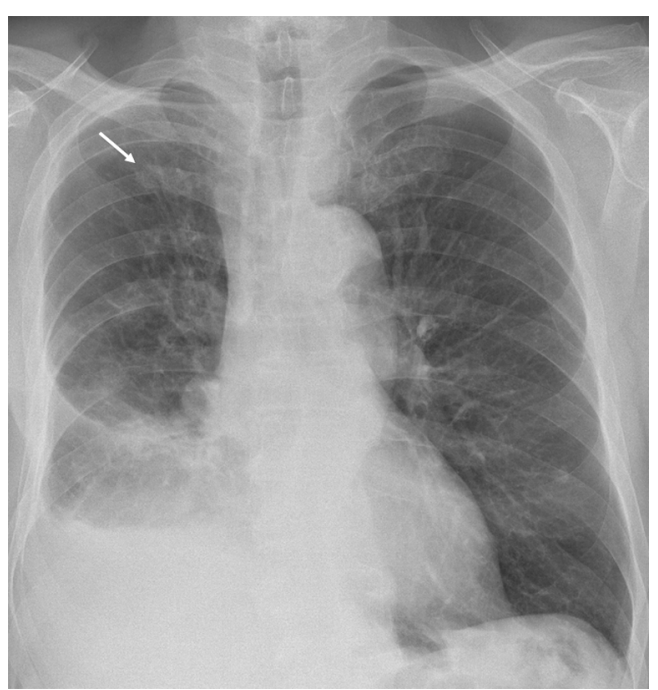

Figure 1 Chest X-ray on admission showing unilateral pleural effusion on the right side and a solitary pulmonary nodule in the right upper lobe (white arrow).

symptoms. On examination, the patient presented thoracic pressure pain on the right, lowered breath sound and subdued resonance over the basal right lung. Confirmatory chest X-ray showed a unilateral pleural effusion on the right side (Figure 1). Initial lab test results revealed normal findings.

For subsequent diagnostic evaluation and therapy, pleural fluid was drained with a chest tube. Cytological and microbiological analysis showed an exudate with negative cytology results and no bacterial growth.

Given the long history of 7 months after thoracic trauma, possible malignancy was considered. Thus, aside from routine diagnostic parameters, CEA and cancer antigen 19-9 (CA 19-9) levels were determined in serum and pleural effusion. Surprisingly, while CA 19-9 levels in serum and pleural fluid was normal, CEA levels were elevated 215 -fold over threshold (reference range $<4.6 \mu \mathrm{g} / \mathrm{L}$ ) in pleural effusion and 30-fold in serum (Figure 2).

Computed tomography (CT) scan of the chest and abdomen revealed a $15-\mathrm{mm}$ pulmonary mass in segment 1 of the right upper lobe (Figure 3).

Due to limited lung reserve, video-assisted thoracoscopic surgery (VATS) wedge resection was performed instead of lobectomy. Besides, multiple pleural biopsies were taken from areas macroscopically suspicious of malignancy. Histopathological analysis showed a $15-\mathrm{mm}$, moderately differentiated acinar cell adenocarcinoma without infiltration

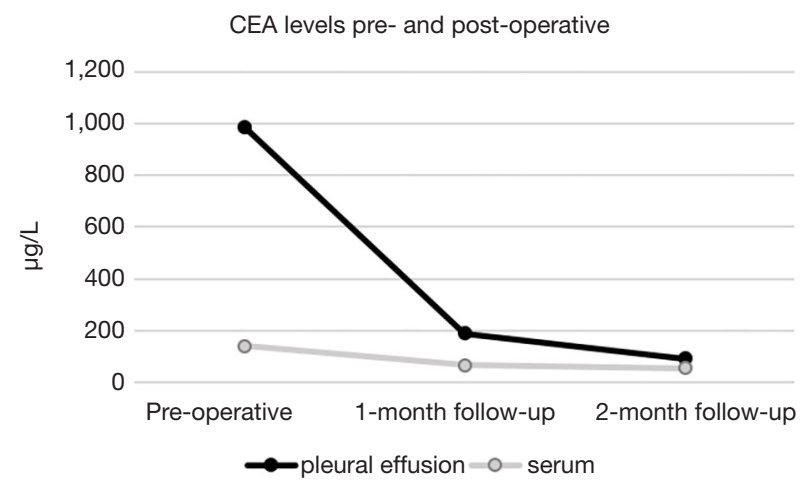

Figure 2 CEA concentration in $\mu \mathrm{g} / \mathrm{L}$, reference range $<4.6 \mu \mathrm{g} / \mathrm{L}$. Serum and pleural CEA concentration measured preoperatively and for follow-up at 1 and 2 months, depicting declining CEA levels after tumor removal. CEA, carcinoembryonic antigen.

of the visceral pleura (pT1b cN0 cM0 R0) (Figure 4). Analysis of specimens obtained from parietal pleura revealed low grade chronic fibrosing pleuritis with no sign of tumor infiltration.

CEA levels in serum were determined 2 and 3 weeks postoperatively as well as during follow-up after 1 and 2 months. Serum and pleural CEA levels showed an overall decline of CEA concentrations up to now at 2 months follow-up (Figure 2).

\section{Discussion}

This case reveals a previously unencountered constellation of early stage pulmonary adenocarcinoma which has not affected visceral or parietal pleura, presenting with cytologically negative pleural effusion, but elevated CEA levels in pleural fluid and serum.

While the infiltration of the visceral pleura explains CEA elevation in malignant pleural effusions associated with lung adenocarcinoma, CEA elevation in this case with negative cytology and absence of pleural affection is not plausible and thus unexpected.

In an early stage tumor as in this case (T1b), the occurrence of malignant pleural effusion is not expected. Considering possible causes for pleural effusion in our case, malignant or benign, increased CEA levels in pleural fluid indicate malignant genesis. However, negative cytological testing indicates lack of circulating tumor cells and histopathology showed no sign of pleural infiltration as well as adequate safety margins after tumor removal (R0). 

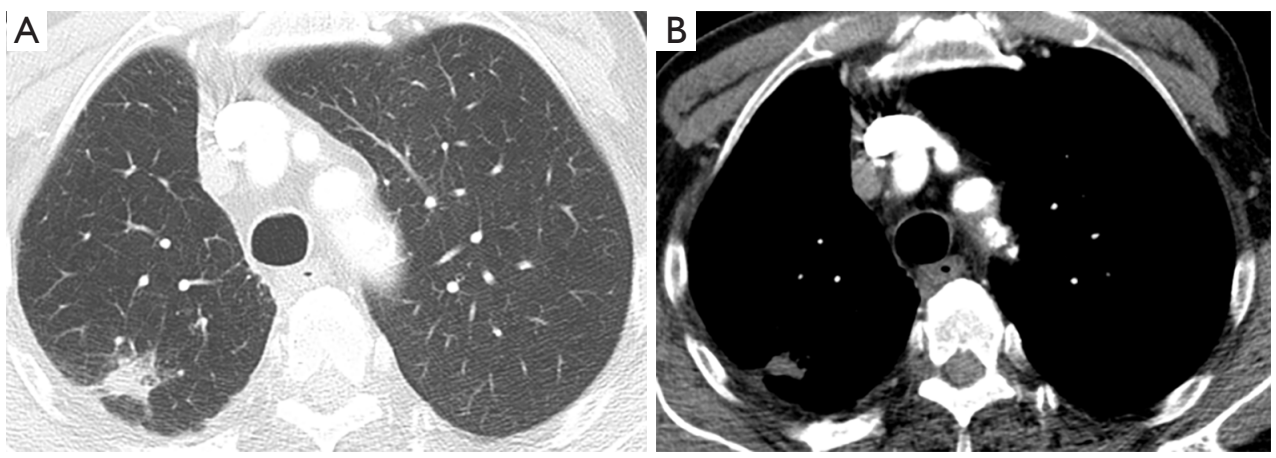

Figure 3 Preoperative CT. (A) Chest CT lung window scanning image showing a $15-\mathrm{mm}$ tumor in the right upper lobe; (B) tumor displayed in chest CT soft tissue window. CT, computed tomography.
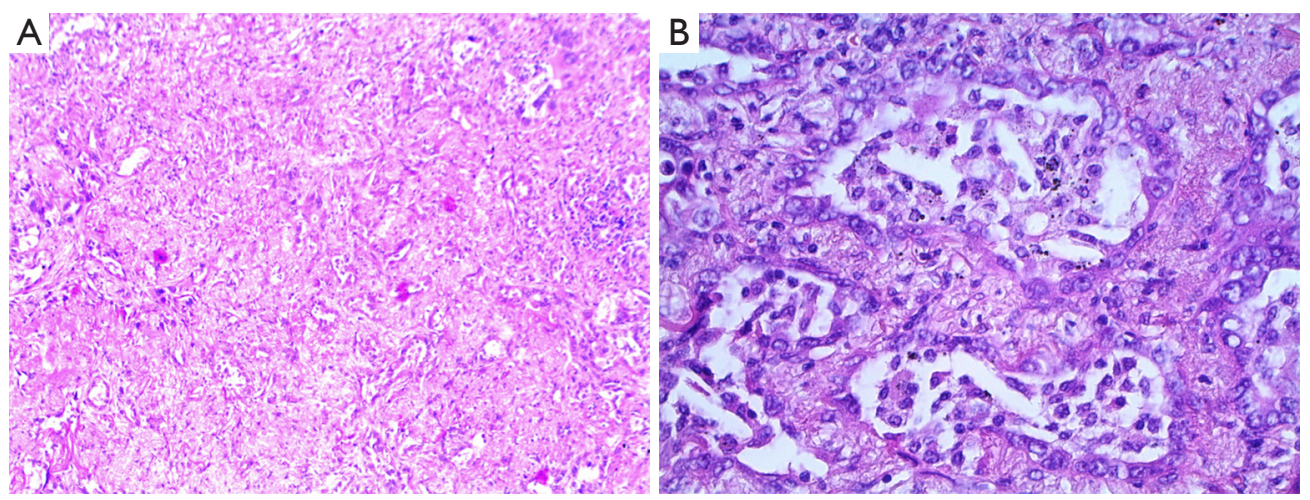

Figure 4 Histopathological image of the analyzed tumor specimen obtained from the right upper lobe by wide wedge resection (HE staining) showed moderately differentiated acinar cell adenocarcinoma (A: ×100; B: ×200).

The patient's history with thoracic trauma and fractured ribs supports the finding of a benign pleural effusion. Additionally, histological examination of parietal pleura biopsies showed fibrous, inflammatory reaction of tissue, but no signs or evidence of malignancy. This raises the question for the causes of the increase of pleural CEA levels in lung adenocarcinoma without pleural affection and cytological examination providing no evidence of tumor cells. One possible explanation is a CEA production by isolated tumor cells circulating in pleural fluid that evade detection in cytological analysis. Unsatisfactory sensitivity of cytological detection of tumor cells in malignant pleural effusions ranging between $30-80 \%(2-6)$ is in support of this hypothesis. Thus, CEA might be an additional indicator of tumor cells present in pleural effusion. Tozzoli et al. showed that pleural CEA levels determined additionally to cytological examination increased the sensitivity in diagnosing malignant pleural effusion in non-small cell lung cancer (NSCLC) to approximately 100\% (2). Another possible hypothesis ist that pleural affection might not have yet occurred. Rather, aside from being secreted by tumor cells, pleural CEA could be elevated due to delayed degradation of CEA caused by slower metabolism in pleural effusion than in serum $(5,7)$.

Review of literature revealed great heterogeneity of data that were included into analysis regarding cytological findings and tumor stages. Pleural effusions seem to be investigated mainly in tumors with pleural infiltration. Hsieh et al. investigated tumor markers in lung adenocarcinomaassociated cytologically negative pleural effusions. These findings showed that mean tumor marker levels were significantly higher in lung adenocarcinoma-associated malignant (cytologically positive) pleural effusion than in cytologically negative pleural effusion, which could indicate that pleural infiltration might have not yet occurred. Nevertheless, positive cytology in all patients is reported 
after 3 months (5).

Moreover, few studies have been published strictly differentiating pleural effusion in lung adenocarcinoma from other histological entities of lung carcinoma. Some studies, however, showed that CEA levels in lung adenocarcinomaassociated pleural effusions are significantly higher than in pleural effusions accompanying other histological entities such as small cell lung cancer (SCLC) and squamous cell carcinoma (1,7-9).

Comparison of serum CEA with pleural CEA levels yielded a positive correlation. Meanwhile, pleural CEA levels appeared to be significantly higher than in serum $(1,2)$. According to this finding, Tozzoli et al. argue against the diagnostic benefit of serum CEA in comparison with determining pleural CEA levels (2). Opposingly, serum CEA level might prove valuable as a follow-up parameter monitoring outcome, relapse or tumor progression.

As implicated by this case report, determining levels of CEA in serum and pleural fluid might prove helpful in certain defined cases. Firstly, in suspected malignant yet cytologically negative pleural effusion the determination of pleural CEA levels leads to a higher diagnostic sensitivity. Secondly, in cases similar to this patient's with incidental and clinically inapparent pulmonary nodules morphologically uncharacteristic for lung cancer, where follow-up via CT imaging is justified. In these cases, the determination of CEA levels in serum and, if present, in pleural effusion, provides a potential tool to rule out or confirm malignancy. This could be especially relevant in patients with pathologically altered lung parenchyma, as in chronic obstructive pulmonary disease (COPD), in which case distinguishing between malignant and benign pulmonary findings can pose a diagnostic challenge. Applied in relevant cases, the diagnostic benefit of CEA level determination highly outweighs the cost, especially with regard to otherwise necessary followup chest CT scans, possible diagnostic VATS or even tumor progression between follow-up examinations.

\section{Acknowledgements}

None.

\section{Footnote}

Conflicts of Interest: The authors have no conflicts of interest to declare.
Informed Consent: Written informed consent was obtained from the patient for publication of this manuscript and any accompanying images.

\section{References}

1. Ogushi F, Fukuoka M, Takada M, et al. Carcinoembryonic antigen (CEA) levels in pleural effusions and sera of lung cancer patients. Jpn J Clin Oncol 1984;14:321-7.

2. Tozzoli R, Basso SM, D'Aurizio F, et al. Evaluation of predictive value of pleural CEA in patients with pleural effusions and histological findings: A prospective study and literature review. Clin Biochem 2016;49:1227-31.

3. Liang QL, Shi HZ, Qin XJ, et al. Diagnostic accuracy of tumour markers for malignant pleural effusion: a metaanalysis. Thorax 2008;63:35-41.

4. Gu Y, Zhai K, Shi HZ. Clinical value of tumor markers for determining cause of pleural effusion. Chin Med J (Engl) 2016;129:253-8.

5. Hsieh TC, Huang WW, Lai CL, et al. Diagnostic value of tumor markers in lung adenocarcinoma-associated cytologically negative pleural effusions. Cancer Cytopathol 2013;121:483-8.

6. Feng $M$, Zhu J, Liang L, et al. Diagnostic value of tumor markers for lung adenocarcinoma-associated malignant pleural effusion: a validation study and meta-analysis. Int J Clin Oncol 2017;22:283-90.

7. Asseo PP, Tracopoulos GD. Simultaneous Enzyme Immunoassay of Carcinoembryonic Antigen in Pleural Effusion and Serum. Am J Clin Pathol 1982;77:66-71.

8. Klech H, Rona G, Hutter C, et al. Diagnostic value of the combined determination of carcinoembryonic antigen (CEA) in pleural effusion and serum with an enzyme immunoassay (EIA). Sensitivity, specificity and relation to tumor type. Wien Klin Wochenschr 1986;98:564-73.

9. Wang XF, Wu YH, Wang MS, et al. CEA, AFP, CA125, CA153 and CA199 in malignant pleural effusions predict the cause. Asian Pac J Cancer Prev 2014;15:363-8.

Cite this article as: Enz N, Fragoso F, Gamrekeli A, Lippek F, Jungraithmayr W. Carcinoembryonic antigen-positive pleural effusion in early stage non-small cell lung cancer without pleural infiltration. J Thorac Dis 2018;10(5):E340-E343. doi: 10.21037/jtd.2018.04.111 